\title{
CCDCGAN: Deep learning prediction of crystal structures
}

\author{
H. Zhang, T. Long, Y. Zhang, N.M. Fortunato, C. Shen, O. Gutfleisch \\ Institute of Materials Science, TU Darmstadt, 64287 Darmstadt, Germany \\ hongbin.zhang@tu-darmstadt.de
}

Autonomous materials discovery with desired properties is one of the ultimate goals for materials science [1]. In this work, we have developed constrained crystal deep convolutional generative adversarial networks (CCDCGAN, Figure 1(a)) based on a proper construction of the latent space [2], which can predict stable crystal structures. In particular, physical properties can be optimized in the latent space, where the formation energy is considered in the current model so that stable structures are predicted directly. We have successfully applied the approach on a randomly chosen binary Bi-Se system and observed that most known phases can be validated with quite a few distinct structures predicted [3]. Furthermore, trained using more than 50,000 compounds in the Materials Project database, we recently extended the algorithm to multicomponent systems coving most elements in the periodic table. As shown in Figure 1(b), two novel structures can be obtained for the Cd-Li system. Detailed analysis reveals that the approach can be used to predict novel crystal structures for various materials systems, and the generation efficiency can be further improved by considering a larger training set. It is expected that the other physical properties (such as band gaps) can be optimized in the latent space as well, giving us the chance to perform multi-objective optimization in the future.

(a)

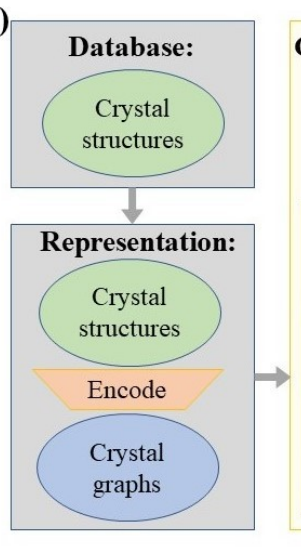

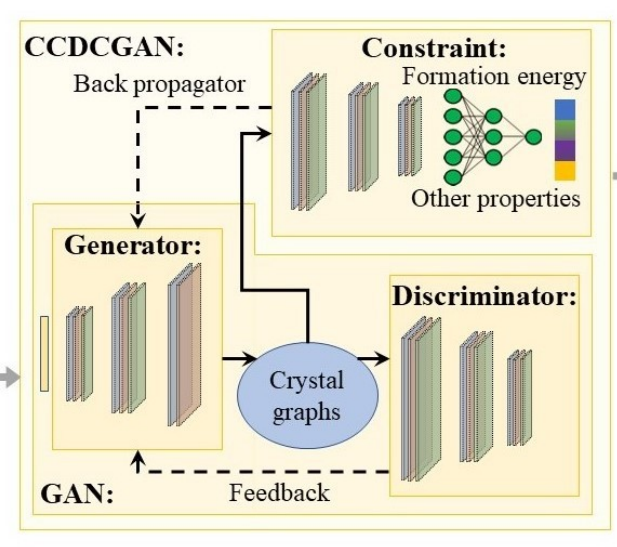
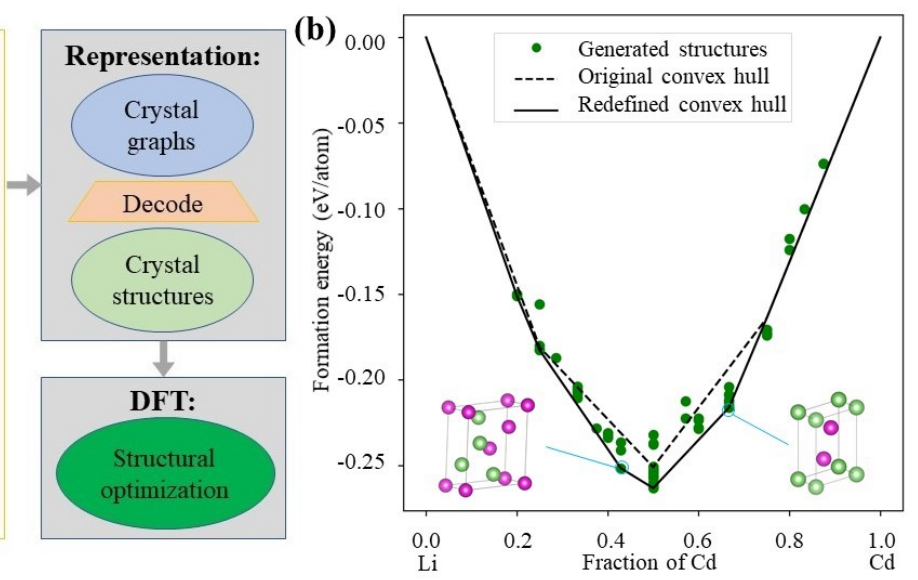

Figure 1. (a) Workflow of CCDCGAN; (b) Convex hull of the Cd-Li system highlighting the predicted structures.

[1] Sanchez-Lengeling, B., \& Aspuru-Guzik, A. (2018). Science 361, 360.

[2] Noh, J., Kim, J., Stein, H. S., Sanchez-Lengeling, B., Gregoire, J. M., Aspuru-Guzik, A., \& Jung, Y. (2019). Matter, 1, 1370.

[3] Long, T., Fortunato, N. M., Opahle, I., Zhang, Y., Samathrakis, I., Shen, C., Gutfleisch, O. \& Zhang, H. (2020). arXiv:2007.11228.

Keywords: crystal structure prediction; deep learning; generative adversarial network; multi-objective optimization

This work was supported by the European Research Council (ERC) under the European Union's Horizon 2020 research and innovation programme [Grant No. 743116-project Cool Innov] and the Deutsche Forschungsgemeinschaft (DFG, German Research Foundation) [Project-ID 405553726 - TRR 270]. Teng Long thanks the financial support from the China Scholarship Council [CSC201806220067]. 